\title{
Ring kinetic theory for tagged-particle problems in lattice gases
}

\author{
R. Brito* and M. H. Ernst ${ }^{\dagger}$ \\ Department of Physics, University of Florida, Gainesville, Florida 32611-2085
}

(Received 28 October 1991)

\begin{abstract}
The kinetic theory for tagged-particle problems in lattice-gas cellular automata is extended beyond Boltzmann's mean-field approximation by including correlated ring-type collisions. This theory provides explicit expressions for the velocity autocorrelation function (VACF) for all times in terms of the ring-collision integral, as well as corrections to the Boltzmann values of the transport coefficients. For times long compared to the mean free time, the ring integral equation yields the phenomenological mode-coupling theory and the long-time tails. For intermediate times it describes a slow transition from initial exponential decay to the long-time tails. At short times the ring kinetic theory is exact. In particular, deviations from the Boltzmann result in the VACF of three-dimensional systems after two time steps are calculated explicitly and compared with computer simulations.
\end{abstract}

PACS number(s): 05.20.Dd, 51.10.+y, 05.60.+w

\section{INTRODUCTION}

In applications of lattice-gas cellular automata (LGCA's) [1] as models for nonequilibrium fluids, almost all theoretical analysis is based on the Boltzmann equation [2]. This is essentially a mean-field theory that neglects the correlations between the occupations of different velocity states. Time correlation functions obtained from it are exact after one time step in all $d$-dimensional models. They are even exact after two time steps in systems larger than two lattice distances in any direction, but the Boltzmann approximation breaks down in most lattice models after three time steps due to the so-called ring collisions. The exponential decay as predicted by the Boltzmann equation is much too rapid in the intermediate- and long-time regimes. At long times simulations done by Frenkel and co-workers $[3,4]$ show a slow algebraic decay of $t^{-d / 2}$, similar to that found by Alder and Wainwright for continuous fluids [5]. The observed long-time tails are in quantitative agreement with the phenomenological mode-coupling theory $[3,6]$, but have never been derived or justified on the basis of a more fundamental kinetic theory for LGCA's.

However, recently the kinetic theory for fluid type LGCA's has been extended by Kirkpatrick and one of the authors [7] to include the correlated ring collisions. Here we use their method to develop an analogous ring kinetic theory for tagged-particle problems. Its main application here is the study of the velocity autocorrelation function (VACF) of a tagged particle at short, intermediate, and long times. This enables us to derive for long times the phenomenological mode-coupling theory.

At intermediate times, recollisions of particles, ignored in the Boltzmann approximation, start to appear at $t=3$, because the minimum time required for two particles to recollide is three time units. However, if the macroscopic periodicity cell, containing the lattice gas, has at least one spatial period equal to two (slab geometry), one observes deviations from Boltzmann even at $t=2$, due to interactions among particles through the boundaries [4]. These excess geometric correlations have been quantitatively explained from the ring collision integral and were found to be in excellent agreement with the computer simulations, as already reported in an earlier publication [8]. The detailed derivation of this result will be presented in this paper.

Why lattice gases in slab geometries? It turns out that the only existing quasi-three-dimensional LGCA fluid with isotropic Euler and Navier-Stokes fluid dynamic equations is the face-centered-hypercubic (FCHC) model [2]. It is a lattice gas contained in a $L \times L \times L \times 2$ slab, embedded in a four-dimensional FCHC lattice.

One of the few existing results, based on the ring kinetic theory, is the breakdown [9] of the Boltzmann equation for calculating transport coefficients in Lorentz-type LGCA's with independent particles, moving in a fixed array of scatterers. In models with backscattering, where the direction of the velocities is reversed upon collision, the diffusion coefficient, calculated from the ring kinetic theory, can be up to $50 \%$ smaller than the Boltzmann diffusion coefficient. This theoretical prediction has been extensively confirmed by computer simulations [9]. A similar reduction of the diffusion coefficient in a LGCA's of interacting particles was very recently reported by Taylor and Boghosian [10].

The paper is organized as follows. In Sec. II we introduce evolution equations of the LGCA. Section III specializes on the short-time behavior of the VACF. In Sec. IV we develop the general kinetic theory for taggedparticle problems. Section V is devoted to the long-time analysis, which leads to the mode-coupling theory. Finally, we include some comments in Sec. VI.

\section{EVOLUTION EQUATIONS}

In LGCA's both space and time are discrete. There are $N$ fluid particles. At integer times $t=0,1,2, \ldots$ their positions $\mathbf{r}$ are at the nodes of a regular $d$-dimensional lattice $\mathcal{L}$. Their velocities belong to the set of nearest- 
neighbor and possibly next-nearest-neighbor lattice vectors $\left\{\mathbf{c}_{i}\right\}$ with $i=0, \ldots, b-1$, where $b$ is called the bit number. The lattice is contained in a periodic box with $L_{\alpha}$ lattice spacings in the $\alpha$ direction $(\alpha=x, y, \ldots, d)$. The number of sites $V$ is then $V=L_{x} L_{y} \cdots L_{d}$. We impose the Fermi exclusion rule, in which no two particles can be at the same node with the same velocity. The occupation numbers $n_{i}(\mathbf{r}, t)$ are equal to 1 if the channel $\mathbf{c}_{i}$ at node $\mathbf{r}$ is occupied at time $t$ and 0 otherwise. The state of a LGCA at time $t$ is fully determined by specifying the set $\left\{n_{i}(\mathbf{r}, t)\right\}$ for all $\mathbf{r}, i$. The dynamics only conserves particle number and momentum, but not energy.

In order to calculate the VACF one needs to include a tagged particle in the system and register its trajectory. The tagged particle behaves as a fluid particle, but carries a tag. As a consequence, if we "switch off" the tag, we would only see the dynamics of the underlying fluid. During collision the tag redistributes among the fluid particles according to certain collision rules. There are many ways to choose such rules (see Ref. [11] for a detailed analysis). Here we only consider the so-called maximally random collision rules, introduced by Frenkel and co-workers in their computer simulations $[3,12]$. In this case the tag redistributes randomly among the output particles at the same node, regardless of any velocity changes from input to output particles. For a completely filled lattice, the tagged particle becomes a random walker on a regular lattice. The time evolution of a LGCA consists of two steps: collision and propagation. Here $\nu_{i}(\mathbf{r}, t)$ denotes the occupation number for the tagged particle at the integer time and $I_{i}$ the collision operator that depends on the collisions between fluid-fluid and fluid-tagged particles. The collision step can be represented as

$$
\nu_{i}^{\prime}(\mathbf{r}, t)=\nu_{i}(\mathbf{r}, t)+I_{i}(n(t), \nu(t)) .
$$

The prime denotes the value of the occupation number at time $t+|\epsilon|$ with $t=0,1,2, \ldots$. After the collision has taken place, the particles are translated to the nearest neighbors. This is the streaming step, which can be represented as

$$
\nu_{i}^{\prime}(\mathbf{r}, t)=\nu_{i}\left(\mathbf{r}+\mathbf{c}_{i}, t+1\right) \equiv S_{i} \nu_{i}(\mathbf{r}, t+1) .
$$

Here $S_{i}$ is the streaming operator which shifts the argument $\mathbf{r}$ to $\mathbf{r}+\mathbf{c}_{i}$. It may also be represented as a $b V \times b V$ matrix $S_{i j}\left(\mathbf{r}, \mathbf{r}^{\prime}\right)=\delta_{i j} \delta\left(\mathbf{r}^{\prime}, \mathbf{r}+\mathbf{c}_{i}\right)$ with $i=0,1, \ldots, b-1$ and $\mathbf{r} \in \mathcal{L}$. Combining these two equations, we get the forward evolution equation with $t=0,1,2, \ldots$ for the tagged particle as

$$
\nu_{i}\left(\mathbf{r}+\mathbf{c}_{i}, t+1\right)=\nu_{i}(\mathbf{r}, t)+I_{i}(n(t), \nu(t)) .
$$

A similar equation exists for the fluid particles, where $\nu$ is replaced by $n$ and $I$ by the appropriate collision operator $\bar{I}$ for fluid particles. In subsequent sections, it will be convenient to also have a backward evolution equation [7], expressing $\nu_{i}(-t-1)$ in terms of $\nu(-t), n(-t)$. As collision rules are invariant under reversal of velocities, the collision step becomes

$$
\begin{aligned}
& \nu_{i}(\mathbf{r},-t)=\nu_{i}^{\prime}(\mathbf{r},-t)+I_{i}\left(n^{\prime}(-t), \nu^{\prime}(-t)\right), \\
& \nu_{i}^{\prime}(\mathbf{r},-t)=\nu_{i}\left(\mathbf{r}+\mathbf{c}_{i},-t+1\right)=S_{i} \nu_{i}(\mathbf{r},-t+1) .
\end{aligned}
$$

The combination of both expressions yields the backward evolution equation $(t>0)$ :

$\nu_{i}(\mathbf{r},-t-1)=S_{i} \nu_{i}(\mathbf{r},-t)+I_{i}(S n(-t), S \nu(-t))$,

where the operator $S$ shifts the argument $\mathbf{r}$ in $n_{j}$ to $\mathbf{r}+\mathbf{c}_{j}$, and where all occupation numbers are taken at the integer values $-t=0,-1,-2, \ldots$. Further analysis of the collision operator $I_{i}$ is now required. As there is at most one tagged particle in the system, $I_{i}$ is linear in the occupation number $\nu$. However, it is a nonlinear function of the occupation numbers $n$, i.e., in a $b$ bits model the collision term $I_{i}$ contains at most $(b-1) n$ 's, each referring to different velocity channels $\mathbf{c}_{j}$. Iterating the evolution equation $t$ times gives an exact series for $\nu_{i}(\mathbf{r}, t)$ expressed as a polynomial of degree $b^{t}-1$ in the occupation numbers, $n_{k}\left(\mathbf{r}^{\prime}, 0\right)$. The collision operator $I_{i}(n, \nu)$ can be expanded in fluctuations around equilibrium by writing

$$
\delta n_{i}(\mathbf{r}, t)=n_{i}(\mathbf{r}, t)-\left\langle n_{i}\right\rangle=n_{i}(\mathbf{r}, t)-f .
$$

Here \langle\rangle denotes an average over an equilibrium ensemble, and $\left\langle n_{i}\right\rangle=f=\rho / b$ is the reduced density $(0 \leq f \leq 1)$. Expansion of the collision operator yields then

$$
\begin{aligned}
I_{i}(n, \nu) & =\sum_{\lambda=1}^{b} \Omega_{i i_{1} \cdots i_{\lambda}}^{(\lambda)} \nu_{i_{1}} \delta n_{i_{2}} \cdots \delta n_{i_{\lambda}} \\
& \equiv \Omega_{i j}^{(1)} \nu_{j}+\Omega_{i}[\nu n \cdots n],
\end{aligned}
$$

where summation convention and the relation $I_{i}(\langle n\rangle,\langle\nu\rangle)=0$ have been used. The second subscript of $\Omega^{(\lambda)}$ is always referring to the tagged particle, while the third, fourth, etc. (if present) refer to fluid particles. Some properties of the $\Omega$ coefficients are as follows:

(i) $\Omega_{i i_{1} i_{2} \cdots i_{\lambda}}^{(\lambda)}$ is symmetric in the labels $\left(i_{2} \cdots i_{\lambda}\right)$ of the fluid particles.

(ii) $\Omega_{i i_{1} \cdots i_{\lambda}}^{(\lambda)}$ vanishes if at least one pair of the indices out of $\left(i_{1} \cdots i_{\lambda}\right)$ is equal.

(iii) $\Omega_{i j}^{(1)}$ is the linearized collision operator that enters in the Boltzmann equation. Furthermore, $\Omega_{i j}^{(1)}$ is symmetric in $i$ and $j$.

In the following we will frequently use the schematic notation $\Omega_{i}$ [] to denote all terms in Eq. (2.7) with $\lambda \geq 2$. To conclude this section we give some equal time correlation functions, which will be needed later on:

$$
\begin{aligned}
\left\langle\nu_{i}(\mathbf{r})\right\rangle & =\langle\nu\rangle=1 / b V \\
\left\langle\nu_{j}(\mathbf{r}) \nu_{i}\left(\mathbf{r}^{\prime}\right)\right\rangle & =\delta_{i j} \delta\left(\mathbf{r}, \mathbf{r}^{\prime}\right)\langle\nu\rangle \\
\left\langle\nu_{j}\left(\mathbf{r}^{\prime}\right) \delta n_{i}(\mathbf{r})\right\rangle & =\delta_{i j} \delta\left(\mathbf{r}, \mathbf{r}^{\prime}\right)\langle\nu\rangle(1-f) \\
\left\langle\delta n_{i}(\mathbf{r}) \delta n_{j}\left(\mathbf{r}^{\prime}\right)\right\rangle & =\delta_{i j} \delta\left(\mathbf{r}, \mathbf{r}^{\prime}\right) \kappa \\
\left\langle\nu_{i}(\mathbf{r}) \nu_{j}\left(\mathbf{r}^{\prime}\right) \delta n_{k}\left(\mathbf{r}^{\prime \prime}\right)\right\rangle & \\
= & \delta_{i j} \delta_{j k} \delta\left(\mathbf{r}, \mathbf{r}^{\prime}\right) \delta\left(\mathbf{r}^{\prime}, \mathbf{r}^{\prime \prime}\right)\langle\nu\rangle(1-f)
\end{aligned}
$$


with $\kappa=f(1-f)$. The two- and three-point correlation functions vanish unless both velocities and positions are the same. These averages can be extended to four-, five-, etc. point correlation functions.

\section{SHORT-TIME BEHAVIOR}

Once we have defined the dynamics of the tagged particle, we can introduce the object of study of this paper, the VACF,

$$
\phi_{x}(t)=\left\langle v_{x}(t) v_{x}(0)\right\rangle,
$$

where $v_{x}(t)$ is the $x$ component of the velocity of the tagged particle at time $t$. In terms of the occupation numbers, the velocity of the tagged particle is $v_{x}(t)=$ $\sum_{\mathbf{r}, i} c_{i x} \nu_{i}(\mathbf{r}, t)$. Therefore Eq. (3.1) can be written as

$$
\phi_{x}(t)=V \sum_{\mathbf{r}, i j} c_{i x} c_{j x}\left\langle\nu_{i}(\mathbf{r}, t) \nu_{j}(\mathbf{0}, 0)\right\rangle
$$

where translational invariance has been used. The diffusion coefficient $D$ is given in terms of the VACF by a Green-Kubo relation

$$
D=\frac{1}{2} \phi_{x}(0)+\sum_{t=1}^{\infty} \phi_{x}(t)
$$

With the help of Eq. (2.8) we can obtain the value at $t=0$ of the VACF as

$$
\phi_{x}(0)=\left\langle v_{x}^{2}(0)\right\rangle=\frac{1}{b} \sum_{i} c_{i x}^{2} \equiv c_{0}^{2}
$$

where $c_{0}^{2}$ is the speed of sound of an athermal LGCA.

We are now interested in the VACF after a few time steps, starting with $t=1$. Using the evolution equation (2.3) and the expansion of the collision operator (2.7), we find

$$
\phi_{x}(1)=V \sum_{\mathbf{r}, i j} c_{i x} c_{j x}\left\langle\nu_{j}(\mathbf{0})\left(S_{i}^{-1} \nu_{i}(\mathbf{r})+S_{i}^{-1} \sum_{\lambda=1}^{b} \Omega_{i i_{1} \cdots i_{\lambda}}^{(\lambda)} \nu_{i_{1}}(\mathbf{r}) \cdots \delta n_{i_{\lambda}}(\mathbf{r})\right)\right\rangle
$$

where all occupation numbers $\nu$ and $\delta n$ are taken at time $t=0$. Next we use translational invariance to let the adjoint operator of $S_{i}^{-1}$ act on $\nu_{j}(\mathbf{0})$, giving $\nu_{j}\left(\mathbf{c}_{i}\right)$. Consider the first term in Eq. (3.5). It yields, on account of Eq. (2.8),

$$
\left\langle S_{i} \nu_{j}(\mathbf{0}) \nu_{i}(\mathbf{r})\right\rangle=\langle\nu\rangle \delta_{i j} \delta\left(\mathbf{r}, \mathbf{c}_{i}\right) .
$$

Similarly the term with $\Omega^{(1)}$ in Eq. (3.5) gives

$$
\left\langle S_{i} \nu_{j}(\mathbf{0}) \Omega_{i i_{1}}^{(1)} \nu_{i_{1}}(\mathbf{r})\right\rangle=\langle\nu\rangle \Omega_{i j}^{(1)} \delta\left(\mathbf{r}, \mathbf{c}_{i}\right) .
$$

Contributions from terms with $\lambda \geq 2$ are vanishing, because all indices $i_{1}, \ldots, i_{\lambda}$ are necessarily different on account of property (ii) below Eq. (2.7). The average of a single $\delta n$ is vanishing. The exact result for the VACF after one time step in matrix notation is then

$$
\phi_{x}(1)=\frac{1}{b} c_{x}\left(\mathbb{1}+\Omega^{(1)}\right) c_{x}
$$

where $c_{x}$ stands for a $b$ vector with components $c_{i x}$ $(i=0,1,2, \ldots, b-1)$. For most of the existing LGCA models such as Frisch-Hasslacher-Pomeau (FHP), FCHC, eight- and nine-bit models, one can prove that $\mathbf{c}$ is an eigenvector of $\Omega^{(1)}$ with eigenvalue $-\omega[11]$. In that case Eq. (3.8) simplifies to

$$
\phi(1)=c_{0}^{2}(1-\omega) .
$$

For tagged-particle models with maximally random collision rules the eigenvalue is [11]

$$
1-\omega=\frac{1-f}{(b-1) f}\left[1-(1-f)^{b-1}\right]
$$

independent of the dimensionality and the collision rules of the underlying LGCA fluid. It was already mentioned in Sec. II that the Boltzmann approximation is obtained by keeping only the term $\Omega^{(1)}$ in the expansion (2.7). Equation (3.8) shows then that Boltzmann is exact for $t=1$. We will show below that the Boltzmann approximation is also exact for $t=2$ if the shortest linear dimension of the lattice is larger than 2 .

Following steps (3.6)-(3.8), i.e., writing the evolution equation $(2.3)$ as $\nu_{i}\left(\mathbf{r}+\mathbf{c}_{i}, t+1\right)=\nu_{i}(\mathbf{r}, t)+\Omega_{i j}^{(1)} \nu_{j}(\mathbf{r}, t)$, we obtain in Boltzmann approximation

$$
\phi_{B}(t)=\frac{1}{b} c_{x}\left(\mathbb{1}+\Omega^{(1)}\right)^{t} c_{x}=c_{0}^{2}(1-\omega)^{t} .
$$

The diffusion coefficient in Boltzmann approximation follows immediately:

$$
D=c_{0}^{2}\left(\frac{1}{\omega}-\frac{1}{2}\right)
$$

Although the Boltzmann approximation predicts the long-time behavior incorrectly (exponential instead of algebraic decay), its value for the diffusion coefficient is very good. The disagreement between Boltzmann and very accurate computer simulations is less than $1 \%$ for all densities (see Ref. [4]).

Next we consider the VACF after two time steps, which requires the evaluation of $\nu_{i}(\mathbf{r}, 2)$. If we use the forward evolution equation (2.3) twice, we generate a huge num- 
ber of terms, on the order $b^{b}$. However, there exists a simpler method to calculate $\phi(2)$ by using stationarity of the equilibrium state, i.e.:

$$
\phi_{x}(2)=V \sum_{\mathbf{r}, i, j} c_{i x} c_{j x}\left\langle\nu_{i}(\mathbf{r}, 1) \nu_{j}(\mathbf{0},-1)\right\rangle .
$$

Using the forward evolution equation (2.3) for $\nu_{i}(\mathbf{r}, 1)$ and the backward Eq. (2.5) for $\nu_{j}(\mathbf{0},-1)$ we generate only about $b^{2}$ terms. If we take property (ii) below Eq. (2.7) into account we find that the only nonvanishing terms are those with the same number of $\delta n$ 's coming from $\nu(\mathbf{0},-1)$ as from $\nu(\mathbf{r}, 1)$, yielding

$$
\begin{aligned}
\phi_{x}(2)=V \sum_{\mathbf{r}, i j} c_{i x} c_{j x}\{ & \left(\delta_{i k}+\Omega_{i k}^{(1)}\right)\left(\delta_{j l}+\Omega_{j l}^{(1)}\right)\left\langle S_{i}^{-1} \nu_{k}(\mathbf{r}) S_{l} \nu_{l}(\mathbf{0})\right\rangle \\
& \left.+\sum_{\lambda=2}^{b} \Omega_{i i_{1} \cdots i_{\lambda}}^{(\lambda)} \Omega_{j j_{1} \cdots j_{\lambda}}^{(\lambda)}\left\langle S_{i}^{-1} \nu_{i_{1}}(\mathbf{r}) \cdots S_{i}^{-1} \delta n_{i_{\lambda}}(\mathbf{r}) S_{j_{1}} \nu_{j_{1}}(\mathbf{0}) \cdots S_{j_{\lambda}} \delta n_{j_{\lambda}}(\mathbf{0})\right\rangle\right\} .
\end{aligned}
$$

The first term gives the Boltzmann value

$$
\phi_{B}(2)=\frac{1}{b} c_{x}\left(\mathbb{1}+\Omega^{(1)}\right)^{2} c_{x}
$$

Next consider the term with $\lambda=2$ in the expansion (3.14), where $i_{1} \neq i_{2}$ and $j_{1} \neq j_{2}$. It contains the average

$$
\begin{aligned}
\left\langle\nu_{i_{1}}(\mathbf{r}\right. & \left.\left.-\mathbf{c}_{i}\right) \delta n_{i_{2}}\left(\mathbf{r}-\mathbf{c}_{i}\right) \nu_{j_{1}}\left(\mathbf{c}_{j_{1}}\right) \delta n_{j_{2}}\left(\mathbf{c}_{j_{2}}\right)\right\rangle \\
= & \kappa\langle\nu\rangle \delta_{i_{1} j_{1}} \delta_{i_{2} j_{2}} \delta\left(\mathbf{r}, \mathbf{c}_{i}+\mathbf{c}_{j_{1}}\right) \delta\left(\mathbf{r}, \mathbf{c}_{i}+\mathbf{c}_{j_{2}}\right) \\
& +\langle\nu\rangle^{2}(1-f)^{2} \delta_{i_{1} j_{2}} \delta_{i_{2} j_{1}} \delta\left(\mathbf{r}, \mathbf{c}_{i}+\mathbf{c}_{j_{1}}\right) \delta\left(\mathbf{r}, \mathbf{c}_{i}+\mathbf{c}_{j_{2}}\right)
\end{aligned}
$$

where Eq. (2.8) has been used. The term containing $\langle\nu\rangle^{2}$ is of relative order $1 / V$ and will be neglected from now on. The Kronecker $\delta$ 's imply $\mathbf{c}_{j_{1}}=\mathbf{c}_{j_{2}}$ for systems larger than two lattice spacings in all directions. However, $\Omega_{j j_{1} j_{2}}^{(2)}$ in (3.14) vanishes if $j_{1}=j_{2}$ [see property (ii)] and the total contribution of the $(\lambda=2)$ term is vanishing. The same argument applies to all terms with $\lambda>2$. Then Eq. (3.15) is the exact expression for $\phi_{x}(2)$ in systems larger than two lattice spacings.

Suppose now that our system is contained in a periodic box with two lattice spacings in one of the directions, and we impose periodic boundary conditions. Then the condition $\delta\left(\mathbf{c}_{j_{1}}, \mathbf{c}_{j_{2}}\right)$ can be satisfied through the periodic boundary. As an example, consider a one-dimensional strip of the FHP model (see Fig. 1), two lattice spacings wide in the $y$ direction. As a consequence of the boundary conditions, points with the same $x$ component are identified: $\mathbf{r}^{\prime}=\mathbf{r} \bmod \left(\mathbf{c}_{a}-\mathbf{c}_{b}\right)$. If we label the velocities $\mathbf{c}_{i}, i=1, \ldots, 6$ we have identified the points

$\mathbf{r}+\mathbf{c}_{2}=\mathbf{r}^{\prime}+\mathbf{c}_{6}$ or $\mathbf{r}+\mathbf{c}_{3}=\mathbf{r}^{\prime}+\mathbf{c}_{5} \quad(\forall \mathbf{r})$

or, equivalently, all pairs that satisfy the equation $\mathbf{c}_{j_{1}}=$ $\mathbf{c}_{j_{2}} \bmod \left(2 \mathbf{e}_{2}\right)$, where $2 \mathbf{e}_{2}=\mathbf{c}_{a}-\mathbf{c}_{b}$ is the spatial period in the $y$ direction. For this case the pairs $\left(\mathbf{c}_{j_{1}}, \mathbf{c}_{j_{2}}\right)$ are $\left(\mathbf{c}_{2}, \mathbf{c}_{6}\right),\left(\mathbf{c}_{6}, \mathbf{c}_{2}\right),\left(\mathbf{c}_{3}, \mathbf{c}_{5}\right),\left(\mathbf{c}_{5}, \mathbf{c}_{3}\right)$. If we define the excess $\mathrm{VACF}$ as

$$
\delta \phi_{\alpha}(t)=\phi_{\alpha}(t)-\phi_{B}(t)
$$

with $\alpha$ running over the Cartesian components $x, y, z, w$, we obtain

$$
\delta \phi_{x}(2)=\frac{\kappa}{b} \sum_{j_{1} j_{2}}\left(\sum_{i} c_{i x} \Omega_{i j_{1} j_{2}}^{(2)}\right)^{2} \delta\left(\mathbf{c}_{j_{1}}, \mathbf{c}_{j_{2}}\right)
$$

where summations are written out explicitly. The terms with $\lambda>2$ can be analyzed similarly, giving $\delta\left(\mathbf{c}_{j_{1}}, \mathbf{c}_{j_{2}}\right) \cdots \delta\left(\mathbf{c}_{j_{1}}, \mathbf{c}_{j_{\lambda}}\right) \delta\left(\mathbf{r}, \mathbf{c}_{i}+\mathbf{c}_{j_{1}}\right)$. The set of $\delta$ 's cannot be satisfied simultaneously since $\mathbf{c}_{j_{1}} \neq \mathbf{c}_{j_{2}} \neq \cdots \neq$ $\mathbf{c}_{j_{\lambda}}$ (see, however, Appendix A). Consequently, Eq. (3.19) becomes an exact expression for $\delta \phi(2)$ [8], where the implicit sum over $j_{1}$ and $j_{2}$ runs over all possible pairs satisfying $\delta\left(\mathbf{c}_{j_{1}}, \mathbf{c}_{j_{2}}\right)=1$ through the periodic boundary. This excess correlation is not a consequence of the dynamics of the LGCA, but purely a geometric effect of the slab geometry. A further illustration of geometric correlations induced by slab geometries is given in Appendix $A$, where the excess VACF is calculated for the quasitwo-dimensional FCHC model, defined on a slab of size $L \times L \times 2 \times 2$. In that case one has apart from (3.19) an additional contribution involving $\Omega^{(3)}$.

The last part of this section is devoted to the calculation of $\delta \phi(2)$ for the quasi-three-dimensional FCHC model, defined in a slab of size $L \times L \times L \times 2$ of the FCHC lattice. This model has been extensively used to simulate three-dimensional lattice gases, because there exist no three-dimensional lattices with the required isotropy of

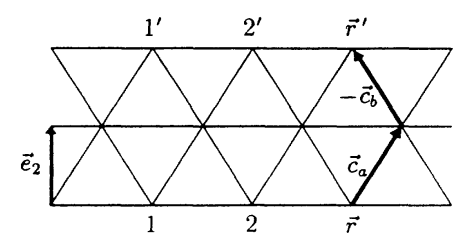

FIG. 1. Quasi-one-dimensional strip of width 2 with periodic boundary conditions $\mathbf{r}^{\prime}=\mathbf{r}+\bmod \left(\mathbf{c}_{a}-\mathbf{c}_{b}\right)$. Points 1 and $1^{\prime}, 2$ and $2^{\prime}$ are identified in slab geometries. 
fourth rank tensors. It consists of $b=24$ allowed velocity states per site $( \pm 1, \pm 1,0,0),( \pm 1,0, \pm 1,0),( \pm 1,0,0, \pm 1)$, $(0, \pm 1, \pm 1,0)$, and $(0,0, \pm 1, \pm 1)$. The essential element in Eq. (3.19) is

$$
\mathbf{A}_{k l} \equiv \sum_{i} \mathbf{c}_{i} \Omega_{i k l}^{(2)} \text {. }
$$

In principle this quantity depends on the detailed collision rules for the tagged particle and the fluid particles. However, for maximally random tagged-particle collision rules (see Sec. II), $\mathbf{A}_{k l}$ has the remarkable property that it is totally independent of the collision rules of the underlying LGCA fluid, similar to the eigenvalue $\omega$ in Eq. (3.10), and for exactly the same reasons [11]. The essential ingredient in the argument is momentum conservation. Let $\{\mathbf{c}\}$ and $\left\{\mathbf{c}^{*}\right\}$, respectively, be the sets of $[p]$ incoming and outgoing velocities in a $p$-tuple collision. Then the expected velocity of the tagged particle after a collision is $(1 / p) \sum_{c^{*}} \mathbf{c}^{*}$. However, because of momentum conservation, $\sum_{c^{*}} \mathbf{c}^{*}=\sum_{c} \mathbf{c}$, independently of the fluid-fluid collision rules. It holds even for noninteracting fluid particles. Hence we can switch off the collisions, and calculate $\mathbf{A}_{k l}$ for a noninteracting fluid. Explicit calculations are given in Appendix B. The result is

$\mathbf{A}_{k l}=\frac{1}{2}\left(\mathbf{c}_{k}+\mathbf{c}_{l}\right) g(f, b)+\frac{1}{2}\left(\mathbf{c}_{k}-\mathbf{c}_{l}\right) h(f, b)$,

with $g$ and $h$ given in terms of the reduced density and the number of bits as

$$
\begin{aligned}
g(f, b)=- & {\left[(b-1)(b-2) f^{2}\right]^{-1} } \\
& \times\left\{2-b f-b(1-f)^{b-1}+(b-2)(1-f)^{b}\right\},
\end{aligned}
$$

$h(f, b)=-[(b-1) f]^{-1}\left\{1-(1-f)^{b-1}\right\}$.

In the FCHC model one can find 12 pairs that satisfy the relation $\delta\left(\mathbf{c}_{j_{1}}, \mathbf{c}_{j_{2}}\right)=1$. But only the 4 pairs with nonzero $x$ component give nonvanishing contributions, which are all equal. This gives a factor 4 in (3.19). Combination of (3.20)-(3.22) gives the exact result for the excess correlation function in the quasi-three-dimensional FCHC model [8]:

$$
\delta \phi_{\alpha}(2)=\frac{1}{6} f(1-f)[g(f, 24)]^{2} \quad(\alpha=x, y, z) .
$$

Similarly we can evaluate the excess correlation function $\delta \phi_{w}$ and the total one, $\phi_{w}(t)$ in Eq. (3.18) for the unphysical fourth dimension $(\alpha=w)$, yielding

$$
\begin{gathered}
\delta \phi_{w}(2)=\frac{1}{2} f(1-f)[h(f, 24)]^{2}, \\
\phi_{w}(2)=\frac{1}{2}(1-\omega)^{2} /(1-f) .
\end{gathered}
$$

The result for $\delta \phi_{x}(2)$ is plotted in Fig. 2 of Ref. [8] as a function of the reduced density $f$. The computer simulations [4] are in a very good agreement with our exact result. The Boltzmann term, also plotted in that figure, shows that for densities $f \gtrsim 0.75$ the corrections (3.23) are the dominant part of $\phi_{x}(2)$. The excess correlation function in the $w$ direction differs from that in the $x, y$, and $z$ directions. The slab geometry of the model breaks the symmetry under interchange of $w \longleftrightarrow \alpha,(\alpha=x, y, z)$, and the tensor $\left\langle v_{\alpha}(t) v_{\beta}(0)\right\rangle$, although still symmetric in $(\alpha, \beta)$, is no longer isotropic. The $(w w)$ component differs from the $(\alpha \alpha)$ component with $\alpha=x, y, z$. As a three-dimensional object, the tensor is still isotropic.

\section{INTERMEDIATE-TIME BEHAVIOR}

In this section we develop the kinetic theory for taggedparticle problems beyond Boltzmann by extending the results for LGCA fluids in Ref. [7]. We will first derive an equation for the two-point propagator of the tagged particle in terms of higher-order correlation functions from which an approximate closed equation can be obtained. Further simplifications yield the one loop or ring diagram, which will be analyzed in Sec. V. We define the kinetic propagator for the tagged particle as

$$
\Gamma_{i j}(\mathbf{r}, t)=b V\left\langle\nu_{i}(\mathbf{r}, t) \nu_{j}(\mathbf{0}, 0)\right\rangle,
$$

normalized as $\Gamma_{i j}(\mathbf{r}, 0)=\delta_{i j} \delta(\mathbf{r}, 0)$ on account of Eq. (2.8). Its Fourier transform is denoted by

$$
\begin{aligned}
\Gamma_{i j}(\mathbf{q}, t) & =\sum_{\mathbf{r}} e^{-i \mathbf{q} \cdot \mathbf{r}} \Gamma_{i j}(\mathbf{r}, t) \\
& =b\left\langle\nu_{i}(\mathbf{q}, t) \nu_{j}^{*}(\mathbf{q}, 0)\right\rangle,
\end{aligned}
$$

with

$$
\Gamma_{i j}(\mathbf{q}, 0)=\delta_{i j} .
$$

The Fourier transforms of the occupation numbers $\nu_{i}$ and $\delta n_{i}$ are defined as

$$
\begin{aligned}
& \nu_{i}(\mathbf{q}, t)=\sum_{\mathbf{r}} e^{-i \mathbf{q} \cdot \mathbf{r}} \nu_{i}(\mathbf{r}, t) \\
& n_{i}(\mathbf{q}, t)=\sum_{\mathbf{r}} e^{-i \mathbf{q} \cdot \mathbf{r}} \delta n_{i}(\mathbf{r}, t)
\end{aligned}
$$

where the following relation holds:

$$
\frac{1}{V} \sum_{\mathbf{r}} e^{-i \mathbf{r} \cdot\left(\mathbf{q}-\mathbf{q}^{\prime}\right)}=\delta\left(\mathbf{q}, \mathbf{q}^{\prime}\right) .
$$

In terms of the propagator, the VACF can be written as

$$
\begin{aligned}
\phi_{x}(t) & =\frac{1}{b} \sum_{\mathbf{r}, i j} c_{i x} \Gamma_{i j}(\mathbf{r}, t) c_{j x} \\
& =\frac{1}{b} \sum_{i j} c_{i x} \Gamma_{i j}(\mathbf{q}=\mathbf{0}, t) c_{j x} .
\end{aligned}
$$

To obtain a kinetic equation for the propagator we write the forward evolution equation (2.3) with (2.7) more schematically as

$\nu_{i}(t+1)=S_{i}^{-1}\left(\mathbb{1}+\Omega^{(1)}\right)_{i j} \nu_{j}(t)+S_{i}^{-1} \Omega_{i}[\nu(t) \cdots n(t)]$,

where $\mathbb{1}$ is the unit matrix and where the node label $\mathbf{r}$ has been suppressed. We will also occasionally use a vector notation for (4.7) by simply dropping the labels $(i, j, \ldots)$, referring to the velocity channels. In fact, the schematic notation (4.7) is valid both in real space as well as in Fourier representation. In the latter $\nu(t)$ represents 
$\nu_{i}(\mathbf{q}, t), S$ represents $S_{i}(\mathbf{q})=\exp (i \mathbf{q} \cdot \mathbf{c})$, and

$$
\begin{aligned}
\Omega_{i}[\nu \cdots n] \equiv \sum_{\lambda=2}^{b} V^{1-\lambda} & \sum_{\substack{\mathbf{q}_{1}, \ldots, \mathbf{q}_{\lambda}\\
}} \delta\left(\mathbf{q}, \sum_{p} \mathbf{q}_{p}\right) \\
& \times \Omega_{i i_{1} \cdots i_{\lambda}}^{(\lambda)} \nu_{i_{1}}\left(\mathbf{q}_{1}, t\right) \cdots n_{i_{\lambda}}\left(\mathbf{q}_{\lambda}, t\right) .
\end{aligned}
$$

We proceed with the solution of (4.7) and consider it as a matrix equation with an inhomogeneity $\mathcal{I}=$ $S^{-1} \Omega[]$. The solution of the homogeneous equation, $\nu(t)=\Gamma^{0}(t) \nu(0)$, involves the kinetic propagator, which reads, respectively, in real space and Fourier representation,

$$
\begin{aligned}
& \Gamma^{0}(\mathbf{r}, t)=\left[S^{-1}\left(\mathbb{1}+\Omega^{(1)}\right)\right]^{t}, \\
& \Gamma^{0}(\mathbf{q}, t)=\left[e^{-i \mathbf{q} \cdot \mathbf{c}}\left(\mathbb{1}+\Omega^{(1)}\right)\right]^{t} .
\end{aligned}
$$

The matrix $\Gamma^{0}$ is in fact the Boltzmann approximation to $\Gamma$. As mentioned before, the streaming operator $S$ may be represented in real space as a $b V \times b V$ matrix with elements $S_{i j}\left(\mathbf{r}, \mathbf{r}^{\prime}\right)=\delta_{i j} \delta\left(\mathbf{r}^{\prime}, \mathbf{r}+\mathbf{c}_{i}\right)$. After Fourier transformation it becomes diagonal in the position labels, i.e., $S(\mathbf{q})=\exp (i \mathbf{q} \cdot \mathbf{c})$ is a $b \times b$ matrix with elements $S_{i j}(\mathbf{q})=\delta_{i j} \exp \left(i \mathbf{q} \cdot \mathbf{c}_{i}\right)$. The formal solution of (4.7) becomes then

$$
\nu(t)=\Gamma^{0} \nu(0)+\Gamma^{0} \otimes S^{-1} \Omega[\nu \cdots n],
$$

where the convolution product is defined as

$$
(f \otimes g)(t)=\sum_{\tau=0}^{t-1} f(t-1-\tau) g(\tau) .
$$

Substitution of Eq. (4.10) into the definition of the propagator (4.2) gives

$$
\begin{aligned}
\Gamma(\mathbf{q}, t) & =b\left\langle\nu(\mathbf{q}, t) \nu^{\dagger}(\mathbf{q}, 0)\right\rangle \\
& =\Gamma^{0}+b \Gamma^{0} S^{-1} \otimes\left\langle\Omega[\nu \cdots n] \nu^{\dagger}(0)\right\rangle,
\end{aligned}
$$

where the $b$ vector $\nu^{\dagger}(\mathbf{q}, 0)$ with components $\nu_{j}^{*}(\mathbf{q}, 0)$, denotes the Hermitian adjoint of $\nu(\mathbf{q}, t)$. We further used the relation $b\left\langle\nu(\mathbf{q}, 0) \nu^{\dagger}(\mathbf{q}, 0)\right\rangle=\mathbb{1}$. The identity (4.12) has the structure of an open hierarchy. It expresses a $(1,1)$-correlation function into a sum of $(\lambda, 1)$-correlation functions $\left\langle\nu_{1}(t) \cdots n_{\lambda}(t) \nu_{j}^{*}(0)\right\rangle$ which can be written as

$$
\left\langle\nu_{1}(t) \cdots n_{\lambda}(t) \nu_{j}^{*}(0)\right\rangle=\left\langle\nu_{1}(0) \cdots n_{\lambda}(0) \nu_{j}^{*}(-t)\right\rangle,
$$

where we have used stationarity of the equilibrium averages. Then we may evaluate $\nu_{j}^{*}(\mathbf{q},-t)$ with $t>0$, using the backward evolution equation (2.5):

$$
\begin{aligned}
\nu(-t-1)= & \left(\mathbb{1}+\Omega^{(1)}\right) S \nu(-t) \\
& +\Omega[S \nu(-t) \cdots S n(-t)] .
\end{aligned}
$$

We solve this equation formally as in (4.9) and (4.10) in terms of $\Gamma^{0 \dagger}(t)=\left[\left(\mathbb{1}+\Omega^{(1)}\right) S\right]^{t}$, and use the result in Eq. (4.12) to further evaluate the propagator $\Gamma(\mathbf{q}, t)$. Here it is convenient to consider the Hermitian adjoint of this formal solution, which reads

$$
\begin{aligned}
\nu^{\dagger}(-t)= & \nu^{\dagger}(0) \Gamma^{0}(t) \\
+ & \sum_{\tau=0}^{t-1} \Omega\left[S^{-1} \nu^{*}(-\tau) \cdots S^{-1} n^{*}(-\tau)\right] \\
& \times \Gamma^{0}(t-\tau-1) .
\end{aligned}
$$

Note that the arguments of the occupation numbers are $\nu(-t)$ and $n(-t)$ and that $\Gamma^{0 \dagger}$ is indeed the Hermitian adjoint of $\Gamma^{0}$, as the matrix $\Omega^{(1)}$ is symmetric [see property (ii) below Eq. (2.7)]. We further introduced the adjoint

$$
\left(\Omega^{\dagger}[S \nu \cdots S n]\right)_{i} \equiv \Omega_{i}\left[S^{-1} \nu^{*} \cdots S^{-1} n^{*}\right] .
$$

Inserting (4.15) into (4.13) and combining it with (4.12) yields again the identity

$\Gamma(t)=\Gamma^{0}+b \Gamma^{0} S^{-1} \otimes\left\langle\Omega[\nu \cdots n] \Omega^{\dagger}[S \nu \cdots S n]\right\rangle \otimes \Gamma^{0}$.

In deriving (4.17) we used the relation

$$
\left\langle\Omega[\nu(0) \cdots n(0)] \nu^{\dagger}(0)\right\rangle=0,
$$

which is a consequence of property (ii) below Eq. (2.7). Equation (4.17) expresses the two-point correlation function in terms of $(\mu, \lambda)$-correlation functions,

$$
\begin{aligned}
\Gamma\left(12 \cdots \lambda ; 1^{\prime} 2^{\prime} \cdots \mu^{\prime} ; t\right) \\
=\left\langle\nu_{1}(t) n_{2}(t) \cdots n_{\lambda}(t)\right. \\
\left.\quad \times\left[S_{1^{\prime}}^{-1} \nu_{1^{\prime}}^{*}(0) S_{2^{\prime}}^{-1} n_{2^{\prime}}^{*}(0) \cdots S_{\mu^{\prime}}^{-1} n_{\mu^{\prime}}^{*}(0)\right]\right\rangle
\end{aligned}
$$

and therefore Eq. (4.17) is not a closed equation for $\Gamma$. However, it can be closed using the Gaussian decoupling scheme introduced in Ref. [7]. In this approximation, all higher-order cumulants are neglected and all correlation functions of order larger than 2 are factorized in terms of two-point correlation functions, yielding a closed equation for the tagged-particle propagator $\Gamma$. For more details we refer to [7]. Within this factorization scheme, and using the properties of the $\Omega$ 's, the $(\lambda, \mu)$ contribution of $\Gamma\left(12 \cdots \lambda ; 1^{\prime} 2^{\prime} \cdots \mu^{\prime} ; t\right)$ in $(4.17)$ is nonvanishing only if $\lambda=\mu$. Furthermore, one has to couple $\nu_{1}(t)$ and $\nu_{1^{\prime}}^{*}(0)$ into the single propagator $\left\langle\nu_{1}(t) \nu_{1^{\prime}}^{*}(0)\right\rangle$. Other couplings, e.g., $\left\langle\nu_{1}(t) \delta n_{2^{\prime}}^{*}(0)\right\rangle$, yield contributions that are $\mathcal{O}(1 / V)$ [see comments below (3.16)]. The remaining $\delta n$ 's can be combined in all possible pairs $\left\langle\delta n_{k}(t) \delta n_{l}(0)\right\rangle$, where the label $k$ belongs to the set $\{12 \cdots \lambda\}$ and the label $l$ to the set $\left\{1^{\prime} 2^{\prime} \cdots \mu^{\prime}\right\}$, yielding $(\lambda-1)$ ! possible combinations. The Gaussian decoupling scheme gives for the simplest term in (4.19) with $\lambda=\mu=2$,

$$
\begin{aligned}
& \left\langle\nu_{i}(\mathbf{q}, t) n_{j}\left(\mathbf{q}_{1}, t\right) \nu_{k}^{*}\left(\mathbf{q}^{\prime}, 0\right) n_{l}^{*}\left(\mathbf{q}_{1}^{\prime}, 0\right)\right\rangle \\
& \quad=\left\langle\nu_{i}(\mathbf{q}, t) \nu_{k}^{*}\left(\mathbf{q}^{\prime}, 0\right)\right\rangle\left\langle n_{j}\left(\mathbf{q}_{1}, t\right) n_{l}^{*}\left(\mathbf{q}_{1}^{\prime}, 0\right)\right\rangle \\
& \quad=\langle\nu\rangle V \delta\left(\mathbf{q}, \mathbf{q}^{\prime}\right) \kappa V \delta\left(\mathbf{q}_{1}, \mathbf{q}_{1}^{\prime}\right) \Gamma_{i k}(\mathbf{q}, t) \bar{\Gamma}_{j l}\left(\mathbf{q}_{1}, t\right),
\end{aligned}
$$


where $\bar{\Gamma}_{j l}(t)$ is the kinetic propagator of a fluid particle, defined in real space and Fourier-space representation as

$$
\begin{aligned}
\bar{\Gamma}_{j l}(\mathbf{r}, t) \kappa & =\left\langle\delta n_{j}(\mathbf{r}, t) \delta n_{l}(\mathbf{0}, 0)\right\rangle, \\
\bar{\Gamma}_{j l}(\mathbf{q}, t) \kappa V & =\left\langle n_{j}(\mathbf{q}, t) n_{l}^{*}(\mathbf{q}, 0)\right\rangle .
\end{aligned}
$$

If we consider only the lowest term in (4.17), with $\lambda=$ $\mu=2$, the Gaussian approximation leads to the following result:

$\Gamma_{i j}(t)=\Gamma_{i j}^{0}+\Gamma_{i m}^{0} S_{m}^{-1} \otimes \Omega_{m i_{1} i_{2}}^{(2)} R_{i_{1} i_{2}, j_{1} j_{2}} \Omega_{l j_{1} j_{2}}^{(2)} \otimes \Gamma_{l j}^{0}$,

where the ring integral $R$ is given by

$$
\begin{aligned}
R_{i_{1} i_{2}, j_{1} j_{2}}(\mathbf{q}, \tau)=\frac{\kappa}{V} \sum_{\mathbf{k}} & \Gamma_{i_{1} j_{1}}(\mathbf{k}, \tau) S_{j_{1}}^{-1}(\mathbf{k}) \\
& \times \bar{\Gamma}_{i_{2} j_{2}}(\mathbf{q}-\mathbf{k}, \tau) S_{j_{2}}^{-1}(\mathbf{q}-\mathbf{k}) .
\end{aligned}
$$

Notice that only terms with $i_{1} \neq i_{2}$ and $j_{1} \neq j_{2}$ appear in Eq. (4.22) because of property (ii) below (2.7). The structure of the ring term in (4.22) is a Boltzmann propagator $\left(\Gamma^{0}\right)$, a collision $(\Omega)$, two particles propagating independently, one tagged $\left(\Gamma S^{-1}\right)$ and one fluid propagator $\left(\bar{\Gamma} S^{-1}\right)$, and finally a recollision $(\Omega)$ followed by a Boltzmann propagator $\left(\Gamma^{0}\right)$. This is the normal structure of the ring collision term in the kinetic theory of continuous fluids [13]. Each factor in the convolution product in (4.22) involves at least one time step, i.e., in $\Gamma_{i j}(t)$ the ring integral is evaluated up to $\tau=t-2$. Then, at $t=2$ the ring integral $R(\mathbf{q}, \tau)$ at $\tau=0$ gives the geometric condition $\delta\left(\mathbf{c}_{j_{1}}, \mathbf{c}_{j_{2}}\right)$ [see also Eq. 3.19)]. Consequently, its contraction with $\Omega_{l j_{1} j_{2}}^{(2)}$ vanishes on account of property (ii) below Eq. (2.7). However, in slab geometries, discussed in Sec. III, the geometric condition can be satisfied through the periodic boundary. In that case (4.22) at $t=2$ gives the exact result discussed in Sec. III. In regular geometries, where all spatial periods are larger than 2 , the ring integral $R(\mathbf{q}, \tau)$ vanishes identically at $\tau=0$, and the prediction of the Boltzmann equation, represented by the first term on the right-hand side of (4.22) is still exact at $t=2$. The ring integral is nonvanishing for $\tau=1,2, \ldots$ and corrections to the Boltzmann value of the VACF appear only after three time steps. For instance, $R(\mathbf{q}, 1)$ reduces to

$$
\begin{aligned}
R_{i_{1} i_{2}, j_{1} j_{2}}(\mathbf{q}, 1)= & \kappa \delta\left(\mathbf{c}_{i_{1}}+\mathbf{c}_{j_{1}}, \mathbf{c}_{i_{2}}+\mathbf{c}_{j_{2}}\right) \\
& \times e^{-i \mathbf{q} \cdot\left(\mathbf{c}_{i_{2}}+\mathbf{c}_{j_{2}}\right)} \Omega_{i_{1} j_{1}}^{(1)} \bar{\Omega}_{i_{2} j_{2}}^{(1)},
\end{aligned}
$$

where the $\delta$ function expresses the ring condition, requiring that the four lattice vectors $\left\{\mathbf{c}_{m}\right\}$ form a closed polygon. The quantity $\bar{\Omega}_{i j}^{(1)}$ is the linearized Boltzmann collision operator for fluid particles. Its explicit form is obtained by a fluctuation expansion similar to (2.7) of the nonlinear collision term $\bar{I}_{i}(n)$ for fluid particles, defined below (2.3). A similar analysis can be done for the higher-order ring terms in (4.17), as has been discussed extensively in Ref. [7]. The result is an obvious general- ization of (4.22), i.e.,

$$
\begin{aligned}
\Gamma_{i j}(t)= & \Gamma_{i j}^{0}+\Gamma_{i m}^{0} S_{m}^{-1} \\
& \otimes \sum_{\lambda=2}^{b} \Omega_{m 12 \cdots \lambda}^{(\lambda)} R_{12 \cdots \lambda, 1^{\prime} 2^{\prime} \cdots \lambda^{\prime}}^{(\lambda)} \Omega_{l 1^{\prime} 2^{\prime} \cdots \lambda^{\prime}}^{(\lambda)} \otimes \Gamma_{l j}^{0},
\end{aligned}
$$

where the ring terms are only nonvanishing for $t \geq 3$. The term $R^{(\lambda)}$ involves a product of $(\lambda-1)$ propagators $\bar{\Gamma}$ for fluid particles and one tagged particle propagator,

$$
\begin{aligned}
& R_{12 \cdots \lambda ; 1^{\prime} 2^{\prime} \cdots \lambda^{\prime}}^{(\lambda)}(\mathbf{q}, t) \\
&=(\lambda-1) !(\kappa / V)^{\lambda-1} \\
& \quad \times \sum_{\mathbf{q}_{1} \cdots \mathbf{q}_{\lambda}} \delta\left(\mathbf{q}, \sum_{p} \mathbf{q}_{p}\right) \Gamma_{11^{\prime}}\left(\mathbf{q}_{1}, t\right) e^{-i \mathbf{q}_{1} \cdot \mathbf{c}_{1^{\prime}}} \\
& \times \prod_{j=2}^{\lambda}\left[\bar{\Gamma}_{j j^{\prime}}\left(\mathbf{q}_{j}, t\right) e^{-i \mathbf{q}_{j} \cdot \mathbf{c}_{j^{\prime}}}\right] .
\end{aligned}
$$

The ring terms with $\lambda>2$ do not contribute at short times $(t \leq 2)$ and long times, although they may be important at intermediate times. The term with $\lambda=2$, expressed in Eq. (4.22), gives the long-time behavior $t^{-d / 2}$, as predicted by the phenomenological mode-coupling theory $[7,3,6]$, as will be discussed in Sec. V. Equation (4.22), with the possible inclusion of higher ring terms $R^{(\lambda)}$, gives a self-consistent equation for the taggedparticle propagator once we know the fluid-fluid propagator $\bar{\Gamma}$. The fluid propagator obeys equations [7] which are completely analogous to (4.17) and (4.22).

Consider the VACF in Eq. (4.6) and insert Eq. (4.22) for the propagator $\Gamma_{i j}(\mathbf{q}=\mathbf{0}, t)$. It follows from Eqs. (3.8)-(3.11) that $\Gamma^{0}(\mathbf{q}=\mathbf{0}, t) \mathbf{c} \equiv g_{0}(t) \mathbf{c}$, where $g_{0}(t)=(1-\omega)^{t}$ is the eigenvalue. The VACF becomes then

$\phi_{x}(t)=c_{0}^{2} g_{0}+b^{-1} g_{0} \otimes A_{x, i j} R_{i j, k l}(\mathbf{0}) A_{x, k l} \otimes g_{0}$,

where $A_{x, k l}$ is defined in (3.20). The first term on the right-hand side equals the Boltzmann value $\phi_{B}(t)$, defined in (3.11). As $\left(g_{0} \otimes g_{0}\right)(t)=t(1-\omega)^{t-1}$ we finally obtain for the VACF at finite $t$ in the ring approximation

$$
\begin{gathered}
\phi_{x}(t)=\phi_{B}(t) \\
+b^{-1} \sum_{\tau=1}^{t-2} \tau(1-\omega)^{\tau-1} A_{x, i j} R_{i j, k l}(\mathbf{0}, t-\tau-1) \\
\times A_{x, k l} .
\end{gathered}
$$

In order to evaluate Eq. (4.28), we need to solve Eq. (4.22) for $\Gamma_{i j}(\mathbf{q}, t)$ and a similar equation for the fluid propagator $\bar{\Gamma}_{i j}(\mathbf{q}, t)$. The higher-order rings (4.25) can be included in (4.27) in a straightforward manner.

As will be shown in Sec. V, the ring integral $R^{(2)}(\mathbf{q}, t)$ has a slow algebraic decay whereas $(1-\omega)^{t}$ has a fast exponential decay with a relaxation time $t_{0}=1 /[-\ln (1-$ $\omega)$ ] on the order of the mean free time. For $t \gg t_{0}$ the ring integral $R(\mathbf{0}, t-\tau-1)$ may be approximated by $R(\mathbf{0}, t)$ and the $\tau$ summation in (4.27) yields $\omega^{-2}$. The long-time 
behavior in the ring approximation is then obtained as

$$
\phi_{x}(t) \simeq\left(b \omega^{2}\right)^{-1} A_{x, i j} R_{i j, k l}(\mathbf{0}, t) A_{x, k l}
$$

In the long-time analysis of Sec. $\mathrm{V}$ we will replace the propagators $\Gamma$ and $\bar{\Gamma}$ in the ring integral (4.23) by their Boltzmann values $\Gamma^{0}$ and $\bar{\Gamma}^{0}$. This implies that the ring kinetic equation is restricted to include only the simple rings. In a separate publication [14] the ring approximation for the stress-stress correlation function, obtained in Ref. [7], will be evaluated numerically for all $t$ and compared with the existing computer simulations.

Next we consider the contribution of the ring integrals (4.25) to the time-dependent diffusion coefficient $D(t)=$ $\sum_{\tau=0}^{t}{ }^{*} \phi(\tau)$, where the asterisk denotes that the term $\tau=0$ has a weight $1 / 2$. For $t \gg 1 /|\ln (1-\omega)|$ the convolution sums in (4.28) can be carried out and with the help of (3.3) and (3.11) one obtains

$$
\begin{aligned}
& D(t) \simeq c_{0}^{2}\left(\frac{1}{\omega}-\frac{1}{2}\right) \\
&+\frac{1}{b \omega^{2}} \sum_{\tau=1}^{t} \sum_{\lambda=2}^{b} A_{12 \cdots \lambda}^{(\lambda)} R_{12 \cdots \lambda, 1^{\prime} 2^{\prime} \cdots \lambda^{\prime}}^{(\lambda)} \\
& \times(\mathbf{0}, \tau) A_{1^{\prime} 2^{\prime} \cdots \lambda^{\prime}}^{(\lambda)},
\end{aligned}
$$

where we have generalized Eq. (3.20) to

$$
A_{12 \cdots \lambda}^{(\lambda)}=\sum_{j} c_{j x} \Omega_{j 12 \cdots \lambda}^{(\lambda)} .
$$

The first term on the right-hand side of (4.30) is the Boltzmann value of the diffusion coefficient. The remaining terms constitute the contributions of the ring collisions. The long-time limit of $D(t)$ (if it exists) gives the diffusion coefficient $D$.

\section{LONG-TIME TAILS}

This section is devoted to an analysis of the ring integral $R(\mathbf{q}, t)$ in Eq. (4.23) at long times and to the derivation of the phenomenological mode-coupling theory, which has been extensively used $[6,12]$ to derive the long-time behavior of correlation function in LGCA's.

We restrict ourselves to the simple ring integral, $R^{0}(\mathbf{q}, t)$, obtained from Eq. (3.18) by replacing the propagators $\Gamma$ and $\bar{\Gamma}$ by their Boltzmann values, i.e.,

$$
\begin{aligned}
& \Gamma^{0}(\mathbf{q}, t)=\left[\Gamma^{0}(\mathbf{q}, 1)\right]^{t}=\left[e^{-i \mathbf{q} \cdot \mathbf{c}}\left(\mathbb{1}+\Omega^{(1)}\right)\right]^{t}, \\
& \bar{\Gamma}^{0}(\mathbf{q}, t)=\left[\bar{\Gamma}^{0}(\mathbf{q}, 1)\right]^{t}=\left[e^{-i \mathbf{q} \cdot \mathbf{c}}\left(\mathbb{1}+\bar{\Omega}^{(1)}\right)\right]^{t},
\end{aligned}
$$

where $\Omega^{(1)}$ is the linearized Boltzmann collision operator for a tagged particle, introduced in Eq. (2.7), and $\bar{\Omega}^{(1)}$ is the corresponding Boltzmann collision operator for a fluid particle.

To study the time dependence of $R^{0}(\mathbf{q}, t)$ it is convenient to decompose $\Gamma^{0}(\mathbf{q}, t)$ and $\bar{\Gamma}^{0}(\mathbf{q}, t)$ into eigenfunctions and eigenvalues. To do so we first consider the eigenvalue problem for the propagator $\bar{\Gamma}^{0}(\mathbf{q}, 1)$ of a fluid particle in Eq. (5.1), using a matrix notation,

$$
e^{-i \mathbf{q} \cdot \mathbf{c}}\left(\mathbb{1}+\bar{\Omega}^{(1)}\right) a^{\mu}(\mathbf{q})=e^{z_{\mu}(\mathbf{q})} a^{\mu}(\mathbf{q}) .
$$

Here $a_{i}^{\mu}(\mathbf{q})(i=1,2, \ldots, b)$ are the components of the right eigenvector $a^{\mu}(\mathbf{q})$ and $\exp \left[z_{\mu}(\mathbf{q})\right]$ is the corresponding eigenvalue, labeled by $\mu(\mu=1,2, \ldots, b)$. The matrix $e^{-i \mathbf{q} \cdot \mathbf{c}}(\mathbb{1}+\Omega)$ is not symmetric. Therefore the eigenfunctions $\widetilde{a}_{i}^{\mu}(\mathbf{q})$ of the transposed matrix differ from $a^{\mu}(\mathbf{q})$, and one easily verifies that

$$
\widetilde{a}^{\mu}(\mathbf{q})=e^{i \mathbf{q} \cdot \mathbf{c}} a^{\mu}(\mathbf{q}) .
$$

These eigenfunctions form a complete biorthonormal set with normalization and completeness relation, respectively,

$$
\begin{aligned}
\sum_{i} \widetilde{a}_{i}^{\mu}(\mathbf{q}) a_{i}^{\nu}(\mathbf{q}) & =\delta_{\mu \nu} \\
\sum_{\mu} \mathcal{P}_{i j}^{\mu}(\mathbf{q}) & =\sum_{\mu} a_{i}^{\mu}(\mathbf{q}) \widetilde{a}_{j}^{\mu}(\mathbf{q})=\delta_{i j} .
\end{aligned}
$$

The $b \times b$ matrix $\mathcal{P}^{\mu}$ projects a $b$ vector into the $\mu$ th eigenspace. The spectral decomposition of the fluid propagator (5.1) is therefore

$$
\bar{\Gamma}_{i j}^{0}(\mathbf{q}, t)=\sum_{\mu} e^{z_{\mu}(\mathbf{q}) t} \mathcal{P}_{i j}^{\mu}(\mathbf{q}) .
$$

A completely analogous decomposition of the taggedparticle propagator $\Gamma^{0}(\mathbf{q}, t)$ can be made by replacing $\bar{\Omega}^{(1)}$ in Eq. (5.5) by $\Omega^{(1)}$. The eigenvalues and eigenvectors for this case will be denoted by $\exp \left[z_{s}(\mathbf{q})\right]$ and $a_{i}^{s}(\mathbf{q})$ with $s=1,2, \ldots, b$.

Inserting these results in (4.23) yields the spectral decomposition of the simple ring integral

$$
\begin{aligned}
R_{i j, m n}^{0}(\mathbf{q}, t)=\frac{\kappa}{V} \sum_{\mathbf{k}} \sum_{\mu, s} & a_{i}^{s}(\mathbf{k}) a_{j}^{\mu}(\mathbf{q}-\mathbf{k}) \\
& \times \exp \left[z_{s}(\mathbf{k}) t+z_{\mu}(\mathbf{q}-\mathbf{k}) t\right] \\
& \times a_{m}^{s}(\mathbf{k}) a_{n}^{\mu}(\mathbf{q}-\mathbf{k}) .
\end{aligned}
$$

We note that $R^{0}$ is expressed in right eigenvectors only, that the real part of $z_{\mu}(\mathbf{q})$ is always negative, and that the labels $\mu, s$ run over the complete eigenvalue spectra, so that $R^{0}(\mathbf{q}, t)$ in $(5.6)$ is exact.

As we are interested in the long-time behavior, only the slowest decay rates $z_{\mu}(\mathbf{q})$ are of interest. The eigenvalue problem (5.2) for the fluid propagator in the slightly rewritten form

$$
\left[e^{z_{\mu}(\mathbf{q})+i \mathbf{q} \cdot \mathbf{c}}-\mathbb{1}-\bar{\Omega}^{(1)}\right] a^{\mu}(\mathbf{q})=0
$$

is a standard problem in kinetic theory which has been studied for LGCA's in Refs. [15, 16]. The spectrum contains $(d+1)$ slow or hydrodynamic modes, with $\operatorname{Re} z_{\mu}(\mathbf{q})=\mathcal{O}\left(q^{2}\right)$ as $\mathbf{q} \rightarrow 0$, related to number and momentum conservation. There are also $(b-d-1)$ fast or kinetic modes, with $z_{\mu}(\mathbf{q}=0)=-\left|\ln \left(1-\omega_{0}\right)\right|$, where 
$-\omega_{0}$ is a typical eigenvalue of $\bar{\Omega}^{(1)}$, so that $z_{\mu}^{-1}$ is typically on the order of the mean free time $t_{\mathrm{MF}}$ between collisions among fluid particles.

To be specific, there are in athermal LGCA's two sound modes $a_{i}^{\sigma}(\mathbf{q})=\left(c_{0}+\sigma \hat{\mathbf{q}} \cdot \mathbf{c}_{i}\right) / \sqrt{\mathcal{N}_{\sigma}}$ where $z_{\sigma}(\mathbf{q})=$ $-i c_{0} \sigma q-\frac{1}{2} \gamma q^{2}($ as $\mathbf{q} \rightarrow 0)$ with $\sigma= \pm$ and $(d-1)$ shear modes $a_{i}^{l}(\mathbf{q})=\hat{\mathbf{q}}_{\perp l} \cdot \mathbf{c}_{i} /{\sqrt{\mathcal{N}_{l}}}$ where $z_{l}(\mathbf{q})=-\nu q^{2}$ as $\mathbf{q} \rightarrow 0$ with $l=1,2, \ldots, d-1$. We have introduced the set $\hat{\mathbf{q}}=\mathbf{q} / q$ and $\hat{\mathbf{q}}_{\perp l}(l=1,2, \ldots, d-1)$ of orthonormal unit vectors, the kinematic viscosity $\nu$, and the sound damping constant $\gamma$. The normalization constants $\mathcal{N}_{\sigma}=2 b c_{0}^{2}$ and $\mathcal{N}_{l}=b c_{0}^{2}$ are chosen such that $\sum_{i} a_{i}^{\mu}(\mathbf{q}) a_{i}^{\nu}(\mathbf{q})=\delta_{\mu \nu}$ as $\mathbf{q} \rightarrow 0$, compatible with Eq. (5.4). For more details and explicit results we refer the reader to Ref. [15]. In principle there may exist also spurious conservation laws that give rise to slow modes at (large) wave numbers related to the centers of the planes (lines) bordering the Brillouin zone. However, the staggered modes do not couple to the long-time tail of the VACF of a tagged particle [6].

The eigenvalue spectrum of the tagged-particle propagator $\Gamma^{0}$ has only one slow diffusion mode $a_{i}^{s}(\mathbf{q})=$ $\left(\mathcal{N}_{s}\right)^{-1 / 2}=b^{-1 / 2}$ with $z^{s}(\mathbf{q})=-D q^{2}$ as $\mathbf{q} \rightarrow 0$, which is related to conservation of tagged-particle number. The coefficient of self-diffusion $D$ is given in Eq. (3.3). There are also $(b-1)$ fast kinetic modes with $z_{\boldsymbol{s}}(\mathbf{q}=\mathbf{0})=$ $-\left|\ln \left(1-\lambda_{0}\right)\right|$, where $-\lambda_{0}$ is a typical eigenvalue on $\Omega^{(1)}$ and $z_{s}^{-1}$ is typically of the order of the mean free time $t_{0}$ between collisions, suffered by the tagged particle. The times $t_{0}$ and $t_{m f}$ may be very different, depending on the density and on the collision rules.

To determine the long-time behavior $\left(t \gg t_{m f}, t_{0}\right)$ of the ring integral (5.6) we restrict ourselves to the $(d+1)$ hydrodynamic modes, labeled $\mu=\{\sigma= \pm, l=$ $1,2, \ldots, d-1\}$, for the fluid particles and to the single diffusion mode, labeled $s$, for the tagged particle. The slow modes are in both cases linear combinations of collisional invariants, i.e., $\bar{\Omega}^{(1)} a^{\mu}=0$ and $\Omega^{(1)} a^{s}=0$. The contributions to (5.6) of two slow modes with $z_{\mu}(\mathbf{q})+z_{s}(\mathbf{q})=$ $\mathcal{O}\left(q^{2}\right)$ as $\mathbf{q} \rightarrow 0$ yields the slow algebraic decay $t^{-d / 2}$, the so-called long-time tails extensively discussed in the literature [17].

However, Eq. (5.6) does not yet have the precise form of the phenomenological mode-coupling theory [6], used as a starting point to discuss the long-time behavior of tagged-particle correlation functions, such as $\left\langle v_{x}(t) v_{x}(0)\right\rangle$. The subsequent long-time analysis pertains to the long-wavelength components of the tagged-particle propagator $\Gamma_{i j}(\mathbf{q} \rightarrow 0, t)$, in a subspace orthogonal to the diffusive mode $a_{i}^{s}(\mathbf{q})=1 / \sqrt{b},(\mathbf{q} \rightarrow 0)$. For times $t \gg t_{m f}, t_{0}$ the tagged-particle propagator (4.22) can be transformed into

$$
\begin{aligned}
\Gamma_{i j}(\mathbf{q}=\mathbf{0}, t) \simeq & {\left[\left(\mathbb{1}+\Omega^{(1)}\right)^{t}\right]_{i j} } \\
& +\left(1 / \Omega^{(1)}\right)_{i i^{\prime}} \Omega_{i^{\prime} i_{1} i_{2}}^{(2)} R_{i_{1} i_{2}, j_{1} j_{2}}^{0}(\mathbf{0}, t) \\
& \times \Omega_{j^{\prime} j_{1} j_{2}}^{(2)}\left(1 / \Omega^{(1)}\right)_{j^{\prime} j}
\end{aligned}
$$

where the same line of arguments was followed as in the transition from (4.27) to (4.28). Also note that the first term on the right-hand side of (5.8) decays exponentially, and can therefore be neglected.

To further evaluate (5.8) we substitute (5.6) and use identity (C6),

$$
\Omega_{i j k}^{(2)} a_{j}^{s} a_{k}^{\mu}=-\frac{1}{f} \Omega_{i j}^{(1)} a_{j}^{s} a_{j}^{\mu},
$$

where $f=\rho / b$ is the reduced density, and $a_{j}^{s}$ and $a_{j}^{\mu}$ are linear combinations of collisional invariants. The identity holds for general LGCA's and general (including maximally random) tagged-particle collision rules. A proof of the identity is given in Appendix C.

The combination of (5.8) and (5.9) yields, for the longtime behavior of the tagged-particle propagator,

$$
\begin{aligned}
\Gamma_{i j}(\mathbf{0}, t) \simeq\left(\frac{1-f}{V f}\right) \sum_{\mathbf{q}, \mu} * & a_{i}^{s}(\mathbf{q}) a_{i}^{\mu}(-\mathbf{q}) \\
& \times \exp \left[z_{s}(\mathbf{q}) t+z_{\mu}(\mathbf{q}) t\right] \\
& \times a_{j}^{s}(\mathbf{q}) a_{j}^{\mu}(-\mathbf{q}),
\end{aligned}
$$

where the asterisk indicates that the $\mu$ sum is restricted to hydrodynamic modes. This formula represents the mode-coupling theory for tagged-particle problems in LGCA's. With the help of Eqs. (3.2), (4.2), and (5.10) we obtain for the long-time behavior of the VACF,

$$
\phi(t)=\frac{1}{V} \sum_{\mu} \sum_{\mathbf{q}}\left|A^{\mu}(\mathbf{q})\right|^{2} e^{\left[z_{s}(q)+z_{\mu}(q)\right] t},
$$

where the amplitudes $A^{\mu}(\mathbf{q})$ are given by

$$
A^{\mu}(\mathbf{q})=\sqrt{\frac{1-f}{b f}} \sum_{j} c_{j x} a_{j}^{s}(\mathbf{q}) a_{j}^{\mu}(-\mathbf{q}) .
$$

Explicit calculation of the amplitude yields

$$
\begin{aligned}
& A^{\sigma}(\mathbf{q})=\sqrt{\frac{1-f}{2 b f}} \sigma c_{0} \hat{q}_{x}, \\
& A^{l}(\mathbf{q})=\sqrt{\frac{1-f}{b f}} c_{0} \hat{q}_{\perp l, x} .
\end{aligned}
$$

The long-time behavior of the VACF follows from (5.11) in the form

$$
\begin{aligned}
\left\langle v_{x}(t) v_{x}(0)\right\rangle & \\
=\frac{(1-f) c_{0}^{2}}{b f V} \sum_{\mathbf{q}} & {\left[\left(\frac{d-1}{d}\right) e^{-(D+\nu) q^{2} t}\right.} \\
& \left.+\frac{1}{d} \cos \left(c_{0} q t\right) e^{-[D+(1 / 2) \gamma] q^{2} t}\right],
\end{aligned}
$$

where the relation $\sum_{i} c_{i x}^{2}=b c_{0}^{2}$ has been used. The first term represents the contribution from the $(d-1)$ shear modes combined with a self-diffusion mode, and gives the dominant long-time tail $t^{-d / 2}[3,6]$. The second term gives the subleading contribution from a sound mode and the self-diffusion mode [18]. The transport coefficients 
are the self-diffusion coefficient $D$, the kinematic viscosity $\nu$, and the sound damping constant $\gamma$. Equation (5.14) is the starting point of the long-time tail calculations in one, two, three [18], and four dimensions [19]. The agreement between simulations and the mode-coupling result (5.14) is in general very good.

\section{CONCLUSIONS}

The main result of this paper can be summarized as follows. We have extended the lattice-gas kinetic theory for tagged-particle problems beyond the Boltzmann equation by including ring collisions [see Eqs. (4.25) and (4.26)]. This enables us to explain several properties of time correlation functions.

(i) The excess correlations (3.18)-(3.24) in the VACF of the three-dimensional FCHC model at short times, which are of purely geometric origin and induced by the slab geometry of the macroscopic periodicity cell. Agreement between theory [8] and simulations [4] is excellent.

(ii) The long-time tail $t^{-d / 2}$ of the VACF, which is derived here directly from the ring kinetic equation for the lattice gas [see Eqs. (5.10) and (5.14)].

(iii) Intermediate-time behavior of the VACF in (4.22)(4.26), that interpolates between the fast exponential decay of the Boltzmann theory and the slow algebraic decay of the long-time tails.

(iv) The deviations (4.30) between the Boltzmann diffusion coefficient and the observed one for $d>2$. In two dimensions, where $D(t) \sim \ln t$ diverges for large $t$ due to the $1 / t$ tail of the VACF, the renormalized diffusion coefficient (4.30) is only meaningful for intermediate-time regimes (say up to 1000 free times; see [20]). In the fluid-type models, discussed here, the renormalization of the Boltzmann or bare diffusion coefficient by ring collisions is small in the intermediate-time regime. However, in model with backscattering (see [9] and [10]) the renormalized diffusion coefficient may be only a factor 2 smaller than its Boltzmann value.

\section{ACKNOWLEDGMENTS}

It is a pleasure to thank J.W. Dufty, D. Frenkel, M. van der Hoef, and G.A. van Velzen for many stimulating and clarifying discussions. R.B. acknowledges support from a DGICYT Project No. PB88-0140 and M.H.E from a NATO Collaborative Research Grant.

\section{APPENDIX A: EXCESS CORRELATIONS IN QUASI-TWO-DIMENSIONAL MODELS}

In this appendix we will calculate the excess velocity autocorrelation function for the FCHC model when the system is contained in a periodic volume of size $L \times L \times$
$2 \times 2$ with two spatial periods equal to 2 . The excess correlation function (3.18) for such system contains not only terms coming from $\Omega^{(2)}$ as in Eq. (3.9), but also from $\Omega^{(3)}$. The $\Omega^{(2)}$ contribution has been analyzed in Sec. III, but the number of contributing pairs is different. Here, condition $\delta\left(\mathbf{c}_{j_{1}}, \mathbf{c}_{j_{2}}\right)$ can be satisfied either through the fourth or the third dimension. The total number of pairs is 24,8 of which give a nonvanishing contribution, which yields an extra factor of 2 in Eq. (3.23). Denoting the contribution from $\Omega^{(3)}$ by $\phi_{x}^{(3)}(2)$, we have

$$
\begin{aligned}
\phi_{x}^{(3)}(2)=V \sum_{\mathbf{r}, i j} & c_{i x} c_{j x} \Omega_{i i_{1} i_{2} i_{3}}^{(3)} \Omega_{j j_{1} j_{2} j_{3}}^{(3)} \\
\times & \left\langle S_{i}^{-1} \nu_{i_{1}}(\mathbf{r}) S_{i}^{-1} \delta n_{i_{2}}(\mathbf{r}) S_{i}^{-1} \delta n_{i_{3}}(\mathbf{r})\right. \\
& \left.\times S_{j_{1}} \nu_{j_{1}}(\mathbf{0}) S_{j_{2}} \delta n_{j_{2}}(\mathbf{0}) S_{j_{3}} \delta n_{j_{3}}(\mathbf{0})\right\rangle
\end{aligned}
$$

On account of the properties of the $\Omega$ 's, the average in Eq. (A1) has to be factorized in pairs, giving

$\frac{2 \kappa^{2}}{b V} \delta_{i_{1} j_{1}} \delta_{i_{2} j_{2}} \delta_{i_{3} j_{3}} \delta\left(\mathbf{r}, \mathbf{c}_{i}+\mathbf{c}_{j_{1}}\right) \delta\left(\mathbf{c}_{j_{1}}, \mathbf{c}_{j_{2}}\right) \delta\left(\mathbf{c}_{j_{2}}, \mathbf{c}_{j_{3}}\right)$

under the summation sign. Equation (A2) is nonvanishing if

$$
\mathbf{c}_{j_{1}}=\mathbf{c}_{j_{2}} \pm 2 \mathbf{e}_{3}, \quad \mathbf{c}_{j_{2}}=\mathbf{c}_{j_{3}} \pm 2 \mathbf{e}_{4}
$$

Both pairs cannot be equal to the same $\mathbf{e}_{n}$ because it would yield $\mathbf{c}_{j_{\alpha}}=\mathbf{c}_{j_{\beta}}$ for a pair $\alpha, \beta$, and then $\Omega^{(3)}$ vanishes. Condition (A3) can be satisfied in the slab of size $L \times L \times 2 \times 2$ by the triplet:

$\mathbf{c}_{j_{1}}=(0,0,1,-1), \quad \mathbf{c}_{j_{2}}=(0,0,1,1), \quad \mathbf{c}_{j_{3}}=(0,0,-1,1)$

and by a triplet with all the signs reversed. Evaluation of $c_{i x} \Omega_{i i_{1} i_{2} i_{3}}^{(3)}$ can be carried out in similar fashion as in Appendix B, but will not be presented here.

\section{APPENDIX B: EVALUATION OF $h$ AND $g$}

This appendix is devoted to the calculation of the factors $g(f, b)$ and $h(f, b)$ appearing in Eq. (3.21). In order to determine the quantity $\mathbf{A}_{k l}$, defined in (3.20), we calculate first

$$
\mathcal{A}(n, \nu)=\sum_{i} \mathbf{c}_{i} I_{i}(n, \nu)
$$

and perform afterwards a fluctuation expansion (2.7) to obtain $\mathbf{A}_{k l}$.

Consider a $[p]$-tuple tagged-particle collision $(p \geq 2)$ in a noninteracting fluid [see below (3.20)]. There are two contributions to the collision operator $I_{i}$ from this collision, a loss term in which a tagged particle in the channel $i$ scatters into other channels, and a gain term, with the opposite effect. They are represented by 


$$
\begin{aligned}
I_{i}^{(p)}(n, \nu)=\{ & -\nu_{i} n([p]-i) \\
& \left.+\frac{1}{p} \sum_{j \in[p]} \nu_{j} n([p]-j)\right\} \bar{n}([b]-[p]),
\end{aligned}
$$

where $n([p]) \equiv \Pi_{i \in[p]} n_{i}$. The factor $\bar{n}([b]-[p])$ guarantees that the channels $[b]-[p]$ are indeed empty in the $p$-tuple collision. The factor $1 / p$ is a consequence of the maximally random collision rules and expresses that on the average a fraction $1 / p$ of the tagged particles is scattered into any velocity channel of the set $[p]$. By considering all possible collisions we obtain

$$
\begin{aligned}
I_{i}(n, \nu)=\sum_{p=2}^{b} \sum_{[p] \subset[b]} *\{ & -\nu_{i} n([p]-i) \\
& \left.+\frac{1}{p} \sum_{j \in[p]} \nu_{j} n([p]-j)\right\} \bar{n}([b]-[p]),
\end{aligned}
$$

where the $[p]$ sum runs over all possible ways to choose the $p$ particles out of $b$ velocities, and the asterisk indicates the constraint that $i \in[p]$.

We multiply (B3) by $\mathbf{c}_{i}$ and sum over $i$. Next we inter- change the order of summations and make the $i$ sum the inner one. Then we interchange the dummy labels $(i, j)$ to find

$$
\begin{aligned}
\mathcal{A}(n, \nu)=\sum_{p=2}^{b} \sum_{[p] \subset[b]} \sum_{i \in[p]}\left[\frac{1}{p} \sum_{\substack{j \in[p] \\
\times \nu_{i} n([p]-i)}} \mathbf{c}_{j}-\mathbf{c}_{i}\right] \\
([b]-[p]) .
\end{aligned}
$$

By interchanging summations again we finally obtain

$$
\begin{aligned}
& \mathcal{A}(n, \nu) \equiv \sum_{i} \mathcal{A}_{i}(n) \nu_{i}, \\
& \mathcal{A}_{i}(n)=\sum_{l=1}^{b-1} \sum_{[l] \subset[b-1]}\left[\frac{1}{l+1} \sum_{j \in[l]} \mathbf{c}_{j}-\frac{l}{l+1} \mathbf{c}_{i}\right] \\
& \times n([l]) \bar{n}([b]-[l]-i) .
\end{aligned}
$$

A fluctuation expansion with $n_{i}=f+\delta n_{i}$, similar to (2.7), defines the coefficients $\mathbf{A}_{i}, \mathbf{A}_{i j}$, etc.,

$$
\mathcal{A}_{i}(n, \nu)=\mathbf{A}_{i} \nu_{i}+\mathbf{A}_{i j} \nu_{i} \delta n_{j}+\cdots,
$$

where summation convention for repeated indices is implied. The first coefficient is $\mathbf{A}_{i}=\mathcal{A}_{i}(f)$. Expanding (B5) to linear order in $\delta n$ gives then

$$
\sum_{m \neq i} \mathbf{A}_{i m} \delta n_{m}=\sum_{l=1}^{b-1} \sum_{[l] \subset[b-1]} \frac{1}{l+1}\left[\sum_{j \in[l]} \mathbf{c}_{j}-l \mathbf{c}_{i}\right]\left[\sum_{m \in[l]} \delta n_{m} f^{l-1}(1-f)^{b-l-1}-\sum_{m \in[b]-[l]-i} \delta n_{m} f^{l}(1-f)^{b-l-2}\right],
$$

where the first term comes from $n([l])$ and the last from $\bar{n}([b]-[l]-i)$. By pulling out the sum over $\delta n_{m}$ we obtain

$$
\begin{aligned}
\sum_{m \neq i} \mathbf{A}_{i m} \delta n_{m}=\sum_{m \neq i} \delta n_{m}\{ & \sum_{l=1}^{b-1} \sum_{[l-1] \subset[b-2]} \frac{1}{l+1}\left[\sum_{j \in[l]} \mathbf{c}_{j}+\mathbf{c}_{m}-l \mathbf{c}_{i}\right] f^{l-1}(1-f)^{b-l-1} \\
& \left.-\sum_{l=1}^{b-1} \sum_{[l] \subset[b-2]} \frac{1}{l+1}\left[\sum_{j \in[l]} \mathbf{c}_{j}-l \mathbf{c}_{i}\right] f^{l}(1-f)^{b-l-2}\right\} .
\end{aligned}
$$

Finally we make the $j$ sum the outer sum, use the relation $\sum_{j} \mathbf{c}_{j}=0$ and obtain

$$
\begin{aligned}
\mathbf{A}_{i m}= & -\sum_{l=1}^{b-1} \sum_{[l-2] \subset[b-3]}\left(\mathbf{c}_{i}+\mathbf{c}_{m}\right) \frac{1}{l+1} f^{l-1}(1-f)^{b-l-1}+\sum_{l=1}^{b-1} \sum_{[l-1] \subset[b-2]}\left(\mathbf{c}_{m}-l \mathbf{c}_{i}\right) \frac{1}{l+1} f^{l-1}(1-f)^{b-l-1} \\
& +\sum_{l=1}^{b-1} \sum_{[l-1] \subset[b-3]}\left(\mathbf{c}_{i}+\mathbf{c}_{m}\right) \frac{1}{l+1} f^{l}(1-f)^{b-l-2}+\sum_{l=1}^{b-2} \sum_{[l] \subset[b-2]} \mathbf{c}_{i} \frac{l}{l+1} f^{l}(1-f)^{b-l-2} .
\end{aligned}
$$

The summand no longer depends on the set variable $[r]$ with $r=l, l-1, l-2$ and the summation may be performed with the help of

$$
\sum_{[a] \subset[b]} 1=\left(\begin{array}{c}
b \\
a
\end{array}\right)
$$

We collect the terms with $\mathbf{c}_{i}$ and $\mathbf{c}_{m}$ in (B9) and define the intermediate variables $\alpha$ and $\beta$ as

$$
\mathbf{A}_{i m}=\alpha \mathbf{c}_{i}+\beta \mathbf{c}_{m}
$$

to obtain

$$
\alpha=(1-f)^{b-2} \sum_{l=0}^{b-3} \frac{1}{(l+1)(l+2)}\left(\begin{array}{c}
b-3 \\
l
\end{array}\right) x^{l}
$$

$$
\beta=\frac{(1-f)^{b-2}}{f} \sum_{l=1}^{b-2} \frac{1}{l+1}\left(\begin{array}{c}
b-3 \\
l-1
\end{array}\right) x^{l}
$$


with $x=f /(1-f)$. After lengthy but straightforward algebra (B12) gives

$$
\begin{aligned}
& \alpha=-\frac{1-f}{(b-1)(b-2) f^{2}} \\
& \times\left[1-(b-1)(1-f)^{b-2}+(b-2)(1-f)^{b-1}\right] \\
& \beta=-\frac{1}{(b-1)(b-2) f^{2}}\left[1-(b-1) f-(1-f)^{b-1}\right]
\end{aligned}
$$

and the result (3.22) follows.
APPENDIX C: DERIVATION OF EQ. (5.9)

Consider a multiple collision involving a tagged particle. The set of $[p]$ input particles, chosen out of $[b]$ scatters into a set $\left[p^{*}\right]$, and the tagged particle, labeled $i \in[p]$, scatters into $i^{*} \in\left[p^{*}\right]$. Then, we can write the collision operator as

$$
\begin{aligned}
I_{i}(n, \nu)=\sum_{p=2}^{b} \sum_{[p] \subset[b]}\{ & -\nu_{i} n([p]-i) \bar{n}([b]-[p]) \\
& \left.+\nu_{i^{*}} n\left(\left[p^{*}\right]-i^{*}\right) \bar{n}\left([b]-\left[p^{*}\right]\right)\right\} .
\end{aligned}
$$

Expansion to linear order in $\delta n$ yields explicit expressions for $\Omega^{(1)}$ and $\Omega^{(2)}$, which are independent of the detailed collision rules:

$$
\begin{aligned}
& I_{i}(n, \nu)= \Omega_{i j}^{(1)} \nu_{j}+\Omega_{i j l}^{(2)} \nu_{j} \delta n_{l}+\cdots=\sum_{p=2}^{b} \sum_{[p] \subset[b]}\left\{-\nu_{i}+\nu_{i^{*}}\right\} f^{p-1}(1-f)^{b-p} \\
&+\sum_{p=2}^{b} \sum_{[p] \subset\{b]}\left\{\left[\begin{array}{cr}
-\nu_{i} \sum_{m \in[p]-i} \delta n_{m}+\nu_{i^{*}} \sum_{m^{*} \in\left[p^{*}\right]-i^{*}} \delta n_{m^{*}}
\end{array}\right](1-f)\right. \\
&\left.+\left[\begin{array}{cc}
\nu_{i} \sum_{m \in[b]-[p]} \delta n_{m}-\nu_{i^{*}} \sum_{m^{*} \in[b]-\left[p^{*}\right]} \delta n_{m^{*}}
\end{array}\right] f\right\} f^{p-2}(1-f)^{b-p-1} .
\end{aligned}
$$

In Eq. (5.9) we need the operators $\Omega^{(s)}(s=1,2)$ acting on products of collisional invariants. We therefore replace $\nu_{i} \Rightarrow a_{i}^{s}=1$ and $\delta n_{i} \Rightarrow a_{i}=\left\{1, \mathbf{c}_{i}\right\}$ with the result

$\Omega_{i j l}^{(2)} a_{j}^{s} a_{l}=\sum_{p=2}^{b} \sum_{[p] \subset[b]}\left\{\left[-\sum_{m \in[p]-i} a_{m}+\sum_{m \in\left[p^{*}\right]-i^{*}} a_{m}^{*}\right](1-f)+\left[\sum_{m \in[b]-[p]} a_{m}-\sum_{m \in[b]-\left[p^{*}\right]} a_{m}^{*}\right] f\right\} f^{p-2}(1-f)^{b-p-1}$.

As $a_{m}$ is conserved in any collision, it follows that

$$
\sum_{m \in[p]} a_{m}=\sum_{m \in\left[p^{*}\right]} a_{m}^{*}
$$

Therefore the second term in (C3) cancels exactly, yielding

$$
\Omega_{i j l}^{(2)} a_{j}^{s} a_{l}=\sum_{p=2}^{b} \sum_{[p] \subset[b]} f^{p-2}(1-f)^{b-p}\left[a_{i}-a_{i}^{*}\right] .
$$

By comparison with the term $\Omega_{i j}^{(1)} \nu_{j}$ in (C2), with $\nu_{j}$ replaced by $a_{j}^{s} a_{j}$, we obtain the desired identity

$$
\Omega_{i j k}^{(2)} a_{j}^{s} a_{k}^{\mu}=-\frac{1}{f} \Omega_{i j}^{(1)} a_{j}^{s} a_{j}^{\mu},
$$

which will be used in the body of the paper to derive the mode-coupling theory.

* Permanent address: Facultad de Ciencias Físicas, Universidad Complutense, 28040-Madrid, Spain.

$\dagger$ Permanent address: Institute for Theoretical Physics, University of Utrecht, P.O.B. 80006, 3508 TA Utrecht, The Netherlands.

[1] Lattice Gas Methods for Partial Differential Equations, edited by G.D. Doolen, reprint volume (Addison-Wesley,
Reading, MA, 1990).

[2] U. Frisch, D. d'Humières, B. Hasslacher, P. Lallemand, Y. Pomeau, and J.P. Rivet, Complex Syst. 1, 649 (1987); see also [1], p. 75 .

[3] D. Frenkel and M.H. Ernst, Phys. Rev. Lett. 63, 2165 (1989).

[4] M.A. van der Hoef and D. Frenkel, Physica D 47, 191 
(1991).

[5] B. J. Alder and T. E. Wainwright, Phys. Rev. A 1, 18 (1970).

[6] T. Naitoh, M.H. Ernst, and J.W. Dufty, Phys. Rev. A 42, 7187 (1990); M.H. Ernst, Physica D 47, 198 (1990).

[7] T.R. Kirkpatrick and M.H. Ernst, Phys. Rev. A 44, 8051 (1991).

[8] R. Brito and M.H. Ernst, Phys. Rev. A 43, 8384 (1991).

[9] M.H. Ernst, G.A. van Velzen, and P.M. Binder, Phys. Rev. A 39, 4327 (1989); G.A. van Velzen and M.H. Ernst, J. Phys. A 22, 4611 (1989); G.A. van Velzen, ibid. 23, 4953 (1990).

[10] W. Taylor IV and B. Boghosian, Phys. Rev. Lett. (to be published).

[11] M.H. Ernst and T. Naitoh, J. Phys. A 24, 2555 (1991).

[12] M. van der Hoef and D. Frenkel, Phys. Rev. A 41, 4277 (1990).
[13] M. H. Ernst and J.R. Dorfman, Physica A 61, 157 (1979).

[14] G. A. van Velzen, R. Brito, and M.H. Ernst, J. Stat. Phys. (to be published).

[15] R. Brito, M.H. Ernst, and T.R. Kirkpatrick, in Discrete Models of Fluid Dynamics, edited by A.S. Alves (World Scientific, Singapore, 1991), p. 198.

[16] L.S. Luo, H. Chen, S. Chen, G.D. Doolen and Y.C. Lee, Phys. Rev. A 43, 7097 (1991).

[17] M.H. Ernst, E. Hauge, and J.M.J. van Leeuwen, J. Stat. Phys. 15, 7 (1976).

[18] T. Naitoh, M.H. Ernst, M. van der Hoef, and D. Frenkel, Phys. Rev. A 44, 2484 (1991), and (unpublished).

[19] M. Dijkstra, M.A. van der Hoef, and D. Frenkel, Europhys. Lett. 17, 39 (1992).

[20] M.A. van der Hoef and D. Frenkel, Phys. Rev. Lett. 66, 1591 (1991); J.A. Leegwater and G. Szamel, ibid. 67, 408 (1991). 